\title{
Vaccination of risk groups in England using the 13 valent pneumococcal conjugate vaccine: economic analysis
}

\author{
Mark H Rozenbaum health economist and infectious disease modeller ${ }^{1}$, Albert Jan van Hoek health \\ economist and infectious disease modeller ${ }^{2}$, Douglas Fleming director ${ }^{3}$, Caroline L Trotter senior \\ research fellow ${ }^{4}$, Elizabeth Miller consultant epidemiologist ${ }^{2}$, W John Edmunds professor of infectious \\ disease modelling ${ }^{5}$
}

'Unit of PharmacoEpidemiology and PharmacoEconomics, Department of Pharmacy, University of Groningen, Antonius Deusinglaan 1, 9713 AV Groningen, Netherlands; ${ }^{2}$ Immunisation, Hepatitis and Blood Safety Department, Health Protection Agency, London, UK; ${ }^{3}$ Birmingham Research Unit of the Royal College of General Practitioners, Birmingham, UK; ${ }^{4}$ School of Social and Community Medicine, University of Bristol, Bristol, UK; ${ }^{5}$ Centre for the Mathematical Modelling of Infectious Diseases, London School of Hygiene and Tropical Medicine, London, UK

\begin{abstract}
Objective To estimate the cost effectiveness of vaccinating people with high risk conditions against invasive pneumococcal disease using the 13 valent pneumococcal conjugate vaccine.

Design Economic evaluation using a cohort model from the perspective of healthcare providers.

Setting England.

Participants People aged 2 years and older at increased risk of invasive pneumococcal disease due to chronic kidney disease; splenic dysfunction; HIV infection; a compromised immune system; chronic heart, liver, or respiratory disease; or diabetes.

Main outcome measures Costs, gains in life years and quality adjusted life years (QALYs), and incremental cost effectiveness ratios.

Results Increasing indirect protection resulting from the vaccination programme of infants using the 13 valent pneumococcal conjugate vaccine means that the burden of disease preventable by targeting high risk groups will diminish in time. Under base case assumptions-that is, no overall impact on non bacteraemic pneumonia in high risk groups and assuming the high risk vaccination programme would be launched two to three years after the infant programme-the incremental cost effectiveness ratio was estimated to be more than $£ 30000$ ( $€ 37216$; $\$ 48210)$ per QALY gained for most risk groups. If, however, the vaccine does not offer protection against non-bacteraemic pneumococcal pneumonia or the vaccine was introduced concomitantly with the infant 13 valent pneumococcal conjugate vaccination programme then vaccinating high risk people would (more) likely be cost effective.
\end{abstract}

Sensitivity analyses showed that the cost effectiveness was particularly sensitive to assumed herd benefits and vaccine efficacy estimates.

Conclusion Under base case assumptions it is unlikely that a pneumococcal vaccination programme aimed at risk groups could be considered cost effective. Uncertainty could be substantially reduced by establishing the effectiveness of the 13 valent pneumococcal conjugate vaccine against non-bacteraemic pneumococcal pneumonia, particularly in at risk groups.

\section{Introduction}

People with certain clinical conditions such as immunocompromised patients and those with chronic heart or lung disease are at increased risk of invasive pneumococcal disease and related mortality. ${ }^{1}$ To prevent disease among these high risk groups many countries recommend vaccination with the 23 valent polysaccharide vaccine, which has been available since the 1980s. Nevertheless, the efficacy and duration of protection of this vaccine is limited, and the antibody response to revaccination is reduced. ${ }^{23}$ The use of conjugated pneumococcal vaccines could potentially overcome the limitations of the 23 valent polysaccharide vaccine. In children the seven valent pneumococcal conjugate vaccine has been shown to be highly effective in preventing invasive pneumococcal disease caused by vaccine related serotypes. ${ }^{4}$ Data on the efficacy in adults, elderly people, and high risk groups are, however, scarce, with most studies focusing on immunogenicity rather than on efficacy. ${ }^{3}$ The limited data on efficacy that are available suggest that pneumococcal conjugate 
vaccines are effective in preventing invasive pneumococcal disease (and possibly pneumonia) in adults and children infected with HIV, a group in whom the 23 valent polysaccharide vaccine is ineffective. ${ }^{56}$ As the pneumococcal conjugate vaccines are more expensive, there is a need to assess whether the use of these vaccines is justified. Such an assessment is complicated by the interaction (at a population level) between a targeted risk based programme and vaccination of children. The introduction of seven valent pneumococcal conjugate vaccine in the infant immunisation programme led to a dramatic decline in incidence of invasive pneumococcal disease due to vaccine serotypes in all age groups (including those in risk groups). ${ }^{7}$ However, these decreases were partly offset by a simultaneous increase in disease caused by non-vaccine serotypes, reducing the impact on overall invasive pneumococcal disease. ${ }^{8}$

In the infant programme in the United Kingdom, as elsewhere, the seven valent pneumococcal conjugate vaccine has recently been replaced by the 13 valent pneumococcal conjugate vaccine. This higher valency vaccine covers six additional serotypes, including the key replacement serotypes 19A and 7F. Similar herd effects for the additional serotypes, as observed for the seven serotypes included in the seven valent pneumococcal conjugate vaccine after its implementation, can be expected in time. However, high risk groups could potentially still benefit from the faster and greater effects of direct vaccination with the 13 valent pneumococcal conjugate vaccine compared with waiting for the indirect benefit from the herd immunity against the vaccine serotypes generated by the infant programme.

We estimated the effectiveness, costs, and cost effectiveness of vaccinating high risk groups in England using the 13 valent pneumococcal conjugate vaccine, taking into account that herd benefits of the current infant 13 valent pneumococcal conjugate vaccine programme will diminish the potential impact of a specific programme for high risk groups over time.

\section{Methods}

We estimated the costs, health benefits, and cost effectiveness of vaccination of high risk groups with the 13 valent pneumococcal conjugate vaccine on top of the current risk based vaccination programme with the 23 valent polysaccharide vaccine. This was done because the existing programme with the 23 valent polysaccharide vaccine is likely be continued despite the potential introduction of a risk based programme using the 13 valent pneumococcal conjugate vaccine. In addition our risk estimates for pneumococcal disease were estimated in the current situation in which a risk based programme using the 23 valent polysaccharide vaccine is already in place (albeit with a low uptake of vaccination).

As infants are already vaccinated with the 13 valent pneumococcal conjugate vaccine, we restricted our analysis to high risk patients aged 2 years and older. The perspective was from that of the National Health Service, as recommended in the United Kingdom. ${ }^{9}$

\section{Model and population}

We developed a cohort model to determine the cost effectiveness of vaccinating specific high risk groups with the 13 valent pneumococcal conjugate vaccine. Groups included in this analysis were based on a recent study among patients admitted to hospital in England with culture confirmed invasive pneumococcal disease, which compared the prevalence of clinical risk factors in the general population with that in patients admitted to hospital with invasive pneumococcal disease. ${ }^{7}$ The study sample comprised 22298 patients admitted to hospital between April 2002 and March 2009 with an admission record in the hospital episode statistics database for England that could be linked with the dataset of the national invasive pneumococcal disease laboratory held at the Health Protection Agency. ${ }^{7}$

In the current analysis we differentiate between people who are immunocompromised, such as those with HIV, asplenia, or splenic dysfunction or who respond poorly to the vaccine, such as people with chronic kidney disease; and those in immunocompetent risk groups such as patients with chronic heart, liver, or respiratory disease and people with diabetes. ${ }^{7}$

The analytical time frame of the study was until 2021 (we assume that after this time the additional benefits of vaccination would be negligible). However, we extrapolated the long term effects of invasive pneumococcal disease over the full lifetime of the participants in each cohort-that is, until death or 100 years.

\section{Incidence of invasive pneumococcal disease and mortality risks}

Using the most recent data available we estimated age group and risk group specific incidences. Firstly, we calculated age specific incidences of invasive pneumococcal disease for the general population, including cases confirmed by polymerase chain reaction and culture from the epidemiological year 2009-10 (in this paper we refer to epidemiological years, which run from July to June, unless stated otherwise). ${ }^{8}$ These incidences were subsequently used to estimate the incidence of invasive pneumococcal disease in high risk people using the prevalence of clinical risk factors among the general population and the prevalence among the linked patients admitted to hospital with invasive pneumococcal disease. ${ }^{7}$ From the same databases we estimated the age specific share of meningitis and empyema to the total invasive pneumococcal disease burden to allow the inclusion of specific costs related to these outcomes. We also obtained age group and risk group specific case fatality ratios for invasive pneumococcal disease from this same study. ${ }^{?}$

\section{Invasive pneumococcal disease sequelae}

Invasive pneumococcal disease may lead to long term sequelae, especially in the case of meningitis. We obtained the risk of different types of sequelae from a recent meta-analysis. ${ }^{9}$ As patients can have multiple sequelae, we assigned all possible combinations on the basis of the prevalence of the individual conditions and reweighted them such that the overall risk to develop any sequela was equal to the pooled prevalence of $31.7 \%$ as estimated by the meta-analysis. We obtained the losses in overall quality adjusted life years (QALYs) using the most severe QALY weight in the combination.

\section{Non-bacteraemic pneumococcal pneumonia}

To assess whether to include an effect of the 13 valent pneumococcal conjugate vaccine on non-bacteraemic pneumococcal pneumonia in the base case we looked at the impact of the seven valent pneumococcal conjugate vaccine on the overall incidence of non-bacteraemic pneumonia in high risk children. For this we obtained the number of episodes of non-specified pneumonia (ICD J18.X, mentioned in any diagnostic code) and the number of deaths for the same cases (within 30 days of admission) for the years 1997-98 up to 2009-10 (data from 2002-03 to 2009-10 were used for deaths) from the hospital episode statistics database in children aged less than 5 years. Next, we divided individual cases into risk or non-risk groups based on the same ICD codes (see appendix 9 in supplementary file) as used for invasive pneumococcal 
disease, and we calculated incidences. An interrupted time series analysis showed that the incidence of pneumonia requiring admission to hospital in non-high risk children aged less than 5 years (that is, those eligible for vaccination) was significantly reduced after the introduction of the seven valent pneumococcal conjugate vaccine, whereas the incidence in high risk children of the same age was not significantly reduced (see appendix 1 in supplementary file). Based on the striking difference between risk and non-risk groups, and the additional uncertainty about the contribution of Streptococcus pneumoniae to non-bacteraemic pneumonia, particularly in high risk children, we decided not to include an overall impact on non-bacteraemic pneumonia in the base case analysis for the high risk groups. We did, however, explore the potential impact of including an effect against non-bacteraemic pneumonia in specific analyses. For this we used the data on age specific incidence for all cause pneumonia for the year 2010 from hospital episode statistics and projected these forward assuming the same incidence as in 2010. Next we assumed that $S$ pneumoniae would be the causal agent in $42 \%$ of the patients in high risk groups admitted to hospital with non-bacteraemic pneumonia on the basis of the results of the two most recent UK studies available. ${ }^{10}{ }^{11} \mathrm{We}$ then assumed that the contribution of the vaccine serotypes to pneumococcal pneumonia would decline in line with the herd effect of the infant vaccination programme on invasive pneumococcal disease.

\section{Indirect effects}

In virtually all countries the introduction of the seven valent pneumococcal conjugate vaccine was followed by a large reduction in invasive pneumococcal disease owing to vaccine serotypes in vaccinated and unvaccinated age groups, with the indirect benefits in some age groups partially offset by a concomitant increase in invasive pneumococcal disease due to non-vaccine serotypes. ${ }^{4}$ This was also the case in the United Kingdom in which the seven valent pneumococcal conjugate vaccine was introduced in September 2006 with a vaccination schedule of 2, 4, and 13 months, and catch-up vaccination for children aged up to 2 years. ${ }^{8}$ In April 2010, the 13 valent pneumococcal conjugate vaccine replaced the seven valent vaccine in the infant vaccination programme.

To predict the future decrease in invasive pneumococcal disease due to vaccine serotypes in unvaccinated age groups, we divided the serotypes into those covered by the seven valent vaccine and those included in the 13 valent vaccine but not in the seven valent pneumococcal conjugate vaccine. In both cases we used age group specific $(2-4,5-14,15-44,45-64$, and $>64$ years $) \mathrm{UK}$ data on incidence of vaccination before and after the introduction of the seven valent vaccine. The prevaccination period included the incidence data for the years 2000-06, whereas the post-vaccination period included data up to four years after the introduction of the vaccine (2006-10). Using the age group specific annual incidence (adjusted for underlying trends in case ascertainment) we fitted a Poisson regression model adjusting for the population size to predict the future reduction in cases of invasive pneumococcal disease due to the vaccine serotypes (see appendix 2 in supplementary file).

We consequently used the predicted annual decrease in vaccine serotypes to predict the incidence of the additional serotypes

(except for serotype 3 , see below) in the 13 valent vaccine - that is, we assumed that the herd effects for the additional serotypes in this vaccine would be similar to those observed for the serotypes in the seven valent vaccine after the introduction of the routine infant vaccination programme using the seven valent pneumococcal conjugate vaccine in $2006 .^{7}$ The only difference was that we delayed the herd effects for the six additional serotypes in the 13 valent vaccine by one year as the introduction of the vaccination programme using the 13 valent pneumococcal conjugate vaccine was not combined with a catch-up programme. This assumption is supported by the most recent data from the Health Protection Agency, which show no indication of any herd effect yet in people aged 5 years and older, 15 months after implementation of the routine infant vaccination programme using the 13 valent pneumococcal conjugate vaccine. ${ }^{12}$ Furthermore, in the Netherlands, where the vaccination programme using the seven valent pneumococcal conjugate vaccine was launched without a catch-up, herd effects were not observed in the first year after implementation in contrast with the United Kingdom. ${ }^{13}$

We did not include serotype replacement effect in the model as we assumed that it would not affect the incremental cost effectiveness ratio because changes in invasive pneumococcal disease due to non-vaccine serotypes are expected to be the same irrespective of the implementation of the risk group programme.

\section{Vaccine efficacy, number of vaccine doses, duration of protection}

Although the efficacy of the seven valent vaccine in healthy infants is well established, the available data for risk groups and adults is scarce, with most studies reporting data on immunogenicity rather than efficacy. ${ }^{3}$ Data on the efficacy of the 13 valent pneumococcal conjugate vaccine is limited ${ }^{12}$; the current licence for the use in infants and children from 6 weeks to 5 years of age and adults aged 50 years and over was based on immunogenicity rather than efficacy data ${ }^{14}$ (see appendix 3 in supplementary file for an overview of available data).

Considering the limited data available, we carried out a formal elicitation of expert opinion on vaccine related variables to construct a probability distribution that represents the experts' knowledge and uncertainty. ${ }^{15}$ The objectives of the elicitation were to estimate the efficacy of the 13 valent pneumococcal conjugate vaccine (against invasive pneumococcal disease and non-bacteraemic pneumococcal pneumonia) and the duration of protection after one dose of the vaccine (as in the base case analysis) or two doses of the vaccine. Importantly, recent data from our group show that the serotype 3 component of the 13 valent pneumococcal conjugate vaccine seems to be ineffective against invasive pneumococcal disease caused by this serotype. ${ }^{16}$ Therefore, in the model we also assumed no protection against disease or carriage for serotype 3 .

Specific details on the method of elicitation can be found in appendix 4 in the supplementary file. Briefly, we asked five members of the Pneumococcal Subcommittee of the Joint Committee on Vaccination and Immunisation to give an estimate for the efficacy of the 13 valent pneumococcal conjugate vaccine in risk groups based on the available efficacy data for the seven valent pneumococcal conjugate vaccine and immunogenicity data for both the seven valent and the 13 valent pneumococcal conjugate vaccines. We used the estimates to create distributions for vaccine effectiveness using the Sheffield elicitation framework. ${ }^{15}$ Final distributions can be found in table $1 \Downarrow$.

\section{Life years and QALY estimates}

As the life expectancy between the general population and high risk groups differs, ${ }^{17}{ }^{18}$ we calculated specific background mortality for people at high risk (and for the general population for validating purposes). Data were gathered from the Royal College of General Practitioners database (including 0.8 million 
patients; more than $1 \%$ of the UK population) over a period of six years (2005 to 2010). We grouped the patients by risk factor (based on Read codes mapped to ICD-9 codes) and calculated the number of person years and deaths in the high risk group. Using these data we calculated background mortality (see appendix 5 in supplementary file). We also calculated the mortality for non-risk groups and validated these against life tables from the Office for National Statistics. ${ }^{19}$

In addition to life years gained we also calculated QALYs gained by vaccination. For patients admitted to hospital for invasive pneumococcal disease, we used losses in QALYs of 0.0079 per case for bacteraemia and 0.0232 per case for meningitis. ${ }^{20} \mathrm{We}$ assumed that non-bacteraemic pneumococcal pneumonia resulted in a QALY loss of 0.006 per case. ${ }^{21}$ In addition to acute losses in QALYs, we also linked specific losses in QALYs to the sequelae due to meningitis based on a Dutch study ${ }^{22}$ (see table 1 for specific losses in QALYs).

\section{Costs}

All costs are reported in pounds sterling at 2009-10 prices. Where necessary we inflated these using the hospital and community health services pay and price index..$^{23}$ As the perspective was from that of the healthcare provider, we included only direct costs. We used recommended procedures to estimate the costs for patients admitted to hospital with invasive pneumococcal disease. The NHS healthcare resource group software was used, which combines procedure codes and ICD-10 diagnostic codes to output the most relevant healthcare resource group code. We subsequently assigned these codes a cost from the National Schedule of Reference Costs for NHS trusts. As the patients included in our analysis are all high risk, we included only those for which it was likely that the invasive pneumococcal disease episode was the main cause for admission to hospital-defined as those patients who had a primary diagnostic code related to an invasive pneumococcal disease code (see appendix 6 in supplementary file). Table 1 displays the costs and probabilities related to invasive pneumococcal disease. The costs of hospital admission for non-bacteraemic pneumococcal pneumonia were based on reference costs for pneumonia. We used the weighted average costs based on the number of non-elective admissions for pneumonia without complications (NHS reference costs code WADZ11C). Patients who had meningitis without sequelae were assumed to have a single outpatient appointment after discharge; we obtained the cost of treatment and care for patients with sequelae after meningitis from a previous cost effectiveness analysis. ${ }^{24}$

The total cost per dose of 13 valent pneumococcal conjugate vaccine was estimated at $£ 56.61$, consisting of the price of the vaccine (£49.10) and administration costs (£7.51).

\section{Scenario and sensitivity analysis}

We carried out univariate, threshold, scenario, and probabilistic sensitivity analyses. In the univariate sensitivity analyses, relevant variables were based on the $5 \%$ and $95 \%$ quantiles to explore the impact of uncertainty around each variable. A threshold analysis was done in which we varied the vaccine price to investigate the effect on the incremental cost effectiveness ratio.

In specific scenario analyses we explored the impact of changes in vaccine efficacy, vaccine waning, delaying the herd effect of the infant vaccination programme using the 13 valent pneumococcal conjugate vaccine, assuming life expectancy of the general population (rather than using the life expectancy of people in high risk groups), and the effect of discounting.
For the probabilistic sensitivity analyses, we generated variables using Monte Carlo sampling, with outcome values generated by running the model 5000 times using Latin hypercube sampling. When quantitative data about uncertainty around variables were available we used $\log$ normal and $\beta$ distributions (see table 1 for specific distributions). When only a single point estimate was available, we assumed a normal distribution with a coefficient of variation of 0.25 . For all the sensitivity analyses it was assumed that the vaccination programme would be launched in 2012-13 (two to three years after the infant programme).

\section{Outcome measures and cost effectiveness analysis}

The simulation model tracks the incidence of invasive pneumococcal disease and non-bacteraemic pneumococcal pneumonia, the number of deaths, costs, QALYs, and life years. We calculated the net costs, life years gained, and QALYs by summing all the costs, life years, and QALYs and calculating the differences for the evaluations with and without vaccination. The incremental cost effectiveness ratio was calculated by dividing the net costs by either the life years gained or QALYs gained. Health effects and cost were both discounted at 3.5\% according to the UK guidelines. ${ }^{25}$ In the analyses we compared the possible impact of vaccination using the 13 valent pneumococcal conjugate vaccine with that of the current situation. Currently, adults aged more than 65 years and people in at risk groups aged 2 years or more are recommended to be vaccinated with the 23 valent polysaccharide vaccine ${ }^{26}$; however, uptake of the vaccine is relatively low, especially in those aged less than 65 years (see appendix 7 in supplementary file). ${ }^{7} \mathrm{We}$ assumed that the 13 valent pneumococcal conjugate vaccine, would be used in addition to the 23 valent polysaccharide vaccine.

Finally, we assumed that the uptake of the 13 valent pneumococcal conjugate vaccine would be similar to the annual influenza programme in the United Kingdom, at $34.5 \%$ in the age group 2-16, 53.6\% in the age group 16-65, and $72.4 \%$ in the age group 65 and older ${ }^{27}$ and that vaccination with the 13 valent pneumococcal conjugate vaccine would be offered irrespective of previous vaccination with the 23 valent polysaccharide vaccine.

\section{Results}

\section{Incidence of invasive pneumococcal disease, vaccine efficacy, indirect effects, and life expectancy}

Among high risk groups the highest incidence of invasive pneumococcal disease was in young people infected with HIV and the lowest in those with chronic heart disease, diabetes, or splenic dysfunction (see appendix 8 in supplementary file for estimated incidence of invasive pneumococcal disease among high risk groups). Table 1 shows the estimates for vaccine efficacy based on the elicitation of expert opinion and the estimated costs associated with different types of invasive pneumococcal disease. Appendix 2 in the supplementary file presents the Poisson regression for invasive pneumococcal disease due to vaccine serotypes after the introduction of the seven valent pneumococcal conjugate vaccine. Finally, appendix 5 in the supplementary file shows the life expectancy for people in high risk groups. 


\section{Total burden in high risk groups}

Without a vaccination programme based on risk groups, but taking into account the likely herd effects of the infant vaccination programme using the 13 valent pneumococcal conjugate vaccine, the model predicts that from 2012-13 to 2020-21 about 1333 cases of invasive pneumococcal disease due to vaccine serotypes would occur in people at high risk (table $2 \Downarrow$ ). This corresponds to a total loss of about 5900 life years or 6200 QALYs (undiscounted). The herd impact of the infant vaccination programme using the 13 valent pneumococcal conjugate vaccine is large; preventing an additional 6200 invasive pneumococcal disease cases due to vaccine serotypes corresponding to an additional 30400 QALYs lost compared with a continuing infant vaccination programme using the seven valent pneumococcal conjugate vaccine.

\section{Impact on budgets}

A risk based vaccination programme would require 4.1 million vaccine doses (assuming the same vaccine uptake as the annual influenza vaccination programme), resulting in a total cost of around $£ 233 \mathrm{~m}$ (of which $£ 202 \mathrm{~m}$ is attributed to the vaccine and the remainder to administration costs). Focusing on specific high risk groups, in whom vaccination would be most cost effective, could reduce the costs substantially. For example, vaccinating people with chronic liver disease would result in a total net cost of $£ 4.6 \mathrm{~m}$. Furthermore, table $3 \Downarrow$ also shows the impact on budgets of assuming a higher coverage among all risk groups ( $80 \%$ uptake) and the impact assuming the same annual coverage as for the 23 valent polysaccharide vaccine (see appendix 7 in supplementary file). If coverage is no higher than that achieved by the 23 valent vaccine, then the impact on budgets would be much reduced, as this programme only achieves poor levels of uptake.

\section{Cost effectiveness}

The base case analysis (excluding a possible impact against non-bacteraemic pneumococcal pneumonia) assumed it would be possible to start vaccinating at risk groups in the epidemiological year 2012-13. Using a threshold of $£ 30000$ for a willingness to pay for a QALY gained,${ }^{25}$ only vaccination of patients with chronic liver disease (table $4 \Downarrow$ ) would be deemed cost effective. People infected with HIV was the second most favourable at risk group, with an incremental cost effectiveness ratio of $£ 61200$ per QALY gained. Vaccinating all other at risk groups would not be considered cost effective, with an incremental cost effectiveness ratio of more than $£ 80000$ per QALY gained.

\section{Impact of time on cost effectiveness}

The expected indirect benefits as a result of the infant vaccination programme limit the direct effect of targeting high risk groups. As a result the cost effectiveness of vaccinating at risk groups decreases over time as indirect benefits accrue. If a programme targeted at high risk groups had been initiated in 2009-10, then vaccinating immune compromised people and people with chronic respiratory disease and HIV infection could also be deemed cost effective (incremental cost effectiveness ratio of $\leq £ 30000$ per QALY). Figure $1 \Downarrow$ shows the impact of time on the incremental cost effectiveness ratio for the years 2009-10 up to 2015-16.

\section{Sensitivity analyses}

Table 4 shows the impact on the incremental cost effectiveness ratio of assuming an overall impact on non-bacteraemic pneumonia. If included, even vaccinating the whole group at increased risk of invasive pneumococcal disease might be considered cost effective, with an incremental cost effectiveness ratio of $£ 17500$ per QALY. Figure $2 \Downarrow$ shows the maximum costs of vaccination for it to be considered cost effective. These costs will decrease with a decreasing net effect of the vaccine in time. In the base case (no overall impact on non-bacteraemic pneumonia) the vaccine costs have to be reduced for all risk groups, except for patients with chronic liver disease, to consider a risk group programme to be cost effective.

The results of the scenario analyses (table $5 \Downarrow$ ) and the univariate sensitivity analysis (fig $3 \Downarrow$ ) show that the predicted herd effects of the infant programme and vaccine efficacy have a large impact on the incremental cost effectiveness ratios. For instance, if there are no herd effects resulting from the additional types now included in the infant vaccination programme then the cost effectiveness of targeting all high risk groups would be reduced from over $£ 180000$ to around $£ 47000$ per QALY gained. Other important factors were the price of the vaccine, the risk and age group specific incidence, and the case fatality ratio. Also, the scenario analysis showed that the additional benefits of a second dose were outweighed by the doubling of the costs.

\section{Probabilistic sensitivity analysis}

Figure $4 \Downarrow$ shows the cost effectiveness acceptability curves for the risk groups in whom the incremental cost effectiveness ratio was less than $£ 100000$ per QALY. It is clear that if the vaccine does not offer protection against non-bacteraemic pneumococcal pneumonia then only vaccinating patients with chronic liver disease is likely to be considered cost effective, but by assuming an overall impact against non-bacteraemic pneumococcal pneumonia, vaccinating any of the at risk groups would probably be cost effective.

\section{Discussion}

Although the herd effects of the infant vaccination programme using the 13 valent pneumococcal conjugate vaccine will in time indirectly protect people at high risk, the burden of preventable pneumococcal disease will remain high during the first years after the introduction of the vaccination programme. Vaccinating all groups at high risk of invasive pneumococcal disease with the 13 valent pneumococcal conjugate vaccine would have a large impact on budgets, therefore targeting specific high risk groups may be more attractive although this would require general practitioners to identify subgroups among those at increased risk. Our analysis shows that unless the 13 valent pneumococcal conjugate vaccine also offers protection against non-bacteraemic pneumococcal pneumonia, vaccination is unlikely to be considered cost effective for most at risk groups. The assumptions about vaccine efficacy and effectiveness, and in particular that against non-bacteraemic pneumococcal pneumonia, had a large impact on our results, and a great deal of uncertainty surrounds these estimates. Although evidence from randomised controlled trials would be preferable to expert opinion, by the time results are available ${ }^{28}$ the potential benefits of vaccinating high risk groups are already largely limited by the expected herd effects.

\section{Strength and weaknesses of the study}

This is the first economic evaluation of vaccination against pneumococcal disease in specific high risk groups using the 13 valent pneumococcal conjugate vaccine. The two most influential variables on the outcome were the assumed herd protection benefits from the infant pneumococcal vaccination 
programme and the vaccine effectiveness against non-bacteraemic pneumococcal pneumonia.

Dynamic models have been used to predict the herd effects of the infant vaccination programme but their reliability critically depends on the structure and underlying assumptions, such as vaccination coverage, difference in case-carrier ratios between serotypes, and the level of competition between vaccine serotypes and non-vaccine serotypes in carriage. ${ }^{18}{ }^{29}$ Hence any such model predictions are subject to considerable uncertainty. Therefore we decided to predict the future herd effects by using Poisson regression models, assuming that the decrease in the additional serotypes (with the exception of serotype 3 ) would be similar to those observed after the introduction of the seven valent pneumococcal conjugate vaccine. Nevertheless, the herd effect for the six additional serotypes in the 13 valent pneumococcal conjugate vaccine might be different from those in the seven valent vaccine owing to differences in carriage, transmissibility, and the potential to cause disease. ${ }^{30}{ }^{31} \mathrm{We}$ also assumed that the herd effects would be similar among high risk and non-high risk groups, as this was previously also observed for invasive pneumococcal disease due to serotypes in the seven valent pneumococcal conjugate vaccine. ${ }^{7}$ However, as the less invasive serotypes primarily affect people at high risk and the additional serotypes included in the 13 valent pneumococcal conjugate vaccine are the more invasive, people at high risk might benefit less from herd effects compared with healthy people. ${ }^{32}$ This may also explain the failure to find a reduction in non-bacteraemic pneumonia in children at high risk compared with healthy children.

Another key assumption was the vaccine efficacy against invasive pneumococcal disease and non-bacteraemic pneumococcal pneumonia. The main reason for not including an effect against non-bacteraemic pneumococcal pneumonia in the base case analysis was that the time series analysis did not show any measurable effect on admissions due to pneumonia in high risk children eligible for vaccination with the seven valent pneumococcal conjugate vaccine, whereas a significant reduction was observed in non-high risk children of the same age. This might be explained by different pathogens (viral or bacterial) causing pneumonia in high risk populations and for those with pneumococcal pneumonia, a different serotype distribution in high risk compared with low risk people. As we had the ability to analyse our surveillance data by whether patients had comorbidities, which would seem essential for deciding on a risk based vaccination programme, our assumption of the effectiveness against non-bacteraemic pneumonia differs from two previous analyses. ${ }^{33}{ }^{34} \mathrm{We}$ do, however, also note that the effect of being in an at risk group on increasing the risk of invasive pneumococcal disease is more noticeable in children than in adults, ${ }^{7}$ which might mean that our assumption of lack of a direct effect of the 13 valent pneumococcal conjugate vaccine on non-bacteraemic pneumonia in adults may be conservative, ${ }^{5}$ yet consistent with the $B M J$ guidelines for economic evaluations. ${ }^{75}$

Finally, we note that the impact of non-bacteraemic pneumococcal pneumonia was high in our analysis despite a relatively low vaccine efficacy being used in combination with a relatively high waning rate (table 1 ).

The cost effectiveness of vaccination depends heavily on the probability of developing disease. In our analysis this was based on the observed odds of invasive pneumococcal disease in risk groups compared with those not in risk groups and the absolute incidence of non-risk group related disease. One of the caveats of the risk factor study was that patients were attributed to risk groups on the basis of the presence of specific discharge codes.
Some of the risk groups might not have been consistently recorded. The odds for people with asplenia were low in the study, with no obvious increased probability of developing disease, resulting in unfavourable incremental cost effectiveness ratios. Although this might be explained by successful prophylaxis by antibiotics or polysaccharide vaccine, it is possible that people with asplenia were poorly recorded. Therefore the cost effectiveness of some of the described risk groups might have been underestimated, although sensitivity analyses showed that our conclusions remained valid when we increased the incidence. Also, the future incidence of pneumococcal disease due to vaccine serotypes may be affected by changes in the epidemiology of viral respiratory tract infections, such as happened with pandemic A/H1N1 2009 influenza. ${ }^{36}$ This caused a noticeable increase in invasive pneumococcal disease in the age groups with the highest incidence of $\mathrm{H} 1 \mathrm{~N} 1$ infection, ${ }^{36}$ and, given the overlap between the risk groups for influenza and invasive pneumococcal disease, selective vaccination of high risk groups with the 13 valent pneumococcal conjugate vaccine might help mitigate the effects of future increases in such viral infections.

\section{Comparison with other studies}

This is the first cost effectiveness analysis of the 13 valent pneumococcal conjugate vaccine focusing specifically on people at increased risk for invasive pneumococcal disease. As far as we know, two other studies have focused on the cost effectiveness of vaccinating non-infant populations. ${ }^{334}$ A main difference is that these studies focused on older adults ( $>50$ years ${ }^{34}$ and $65^{33}$ years), whereas our study specifically focused on risk groups of people aged 2 years and older. Both these studies showed that for these specific age groups a vaccination programme could be considered cost effective, whereas we in the base-case analysis conclude that a vaccination programme in unlikely to be considered cost effective. The main driver for this difference is that in the base case analysis we assumed that the 13 valent pneumococcal conjugate vaccine would not have an overall impact on non-bacteraemic pneumonia. This difference was further driven by the assumption that vaccine would not be effective against serotype 3, as early data from England and Wales suggests that this component of the 13 valent vaccine does not seem to provide direct protection to vaccinated people. ${ }^{16}$ However, this assumption was based on a few cases of invasive disease due to this serotype in children in England and Wales and future data are necessary to answer the outstanding question on the efficacy of this serotype.

Other differences between our study and these two age based studies are that we had detailed data on the risk of disease, the life expectancy of high risk populations, and specific costs per invasive pneumococcal disease episode available, all based on primary data as opposed to estimates from the literature or databases. Furthermore, compared with the Dutch study we were able to explicitly take herd effects into account for the unvaccinated population as recent data has become available that could be used for the prediction of these effects. ${ }^{8}$

We showed in the current study that these herd effects have a major impact on cost effectiveness. It is desirable that specific cost effectiveness studies from a European perspective become available to guide decision making in European countries rather than using cost effectiveness estimates from the United States. Previous decisions to introduce the infant pneumococcal vaccination programmes in European countries largely relied on herd immunity estimates from the United States that were subsequently shown not to be applicable elsewhere. ${ }^{3}$ 


\section{Implications and future research}

We found that the cost effectiveness of the 13 valent pneumococcal conjugate vaccine programme based on risk group will mainly depend on the time of using the vaccine and its effectiveness, in particular against non-bacteraemic pneumococcal pneumonia. Since most countries have replaced the seven valent pneumococcal conjugate vaccine with the 13 valent vaccine, herd effects are likely to decrease the burden of preventable pneumococcal disease over time rendering any additional preventive efforts less cost effective. If the 13 valent vaccine does protect against non-bacteraemic pneumococcal pneumonia in high risk groups the programme may be cost effective if introduced early enough before the full effect of herd immunity is manifested, or if the expected herd immunity effect is less than expected. Policy makers may prefer to delay any decision about the use of the 13 valent vaccine in high risk groups until the results of the trial currently being done in the Netherlands to assess its efficacy against non-bacteraemic pneumonia in elderly people are available. ${ }^{28}$ However, such a wait and see policy would possibly reduce the need for the additional vaccination effort. Another option for governments to consider would be sharing the risk with the manufacturer; on the basis of the uncertainty around the cost effectiveness a price reduction could be negotiated that remains valid until the data on efficacy become available. The implementation of a risk based vaccination programme using the 13 valent pneumococcal conjugate vaccine in the United Kingdom has been considered by the UK Joint Committee on Vaccination and Immunisation, with the final decision being not to introduce such a programme largely dependent on the outcome of our study. ${ }^{37}$ As many other European countries lack the various high quality epidemiological data sources available in the United Kingdom or lack the statistical power owing to their population size to conduct their own analyses this study will also provide them with important evidence. Specific cost effectiveness ratios cannot directly be extrapolated from England to other countries but we believe that the general conclusion is informative for those countries that introduced the 13 valent pneumococcal conjugate vaccine around the same time and have a similar uptake of vaccination. Some European countries are, however, already recommending the 13 valent pneumococcal conjugate vaccine for at risk groups or adults. For example, in Austria and Greece the 13 valent pneumococcal conjugate vaccine is recommended for those aged 50 and older, ${ }^{38}{ }^{39}$ whereas in France, parts of Germany, and Italy the vaccine is being recommended for (specific) risk groups. ${ }^{4041}$

Finally, we note that in addition to considerations about cost effectiveness, decision makers need to estimate carefully the possible uptake of vaccination, considering the potentially large impact on budgets of a risk based vaccination programme using the 13 valent pneumococcal conjugate vaccine.

We thank Stefan Flasche, Julie Robotham, and Petros Pechlivanoglou for useful discussions on the economic evaluation; Helen Johnson for help with the costings; Michelle Barley for her assistance in extracting the Royal College of General Practitioners data on survival; our expert panel for their estimates on vaccine efficacy; and the JCVI subgroup on pneumococcal vaccination for useful comments.

Contributors: JE and EM designed the study. MHR, A-JvH, and JE designed the computer model and carried out the computer simulations and analysis. $\mathrm{MH}, \mathrm{A}-\mathrm{JvH}$, and CT carried out the data analyses under the supervision of EM and JE. DF was responsible for the analysis of the Royal College of General Practitioners data that provided the survival curves by risk groups. MHR, EM, and JE drafted the manuscript. All authors commented on drafts of the manuscript and contributed to the final version. MHR and JE are guarantors of the study.

Funding: AJvH and JE were supported by the UK Department of Health Policy Research Programme (grant No 039/0031). The funder had no part in the design or execution of the study or the analysis and interpretation of the results. The views expressed here are those of the authors and not necessarily those of the Department of Health.

Competing interests: All authors have completed the ICMJE uniform disclosure form at www.icmje.org/coi_disclosure.pdf (available on request from the corresponding author) and declare: no support from any organisation for the submitted work; MHR was involved in this study while an employee at the University of Groningen. MHR joined Pfizer Netherlands on 1 September 2011. Pfizer had no involvement in the development of the model nor any influence on the results, outcomes, and conclusions drawn or in the preparation of this paper; no other relationships or activities that could appear to have influenced the submitted work.

Ethical approval: Not required.

Data sharing: No additional data available.

1 Prevention of pneumococcal disease: recommendations of the Advisory Committee on Immunization Practices (ACIP). MMWR Recomm Rep 1997;46(RR-8):1-24.

2 Huss A, Scott P, Stuck AE, Trotter C, Egger M. Efficacy of pneumococcal vaccination in adults: a meta-analysis. CMAJ 2009;180:48-58.

3 Musher DM, Sampath R, Rodriguez-Barradas MC. The potential role for protein-conjugate pneumococcal vaccine in adults: what is the supporting evidence? Clin Infect Dis 2011;52:633-40.

4 Rozenbaum MH, Boersma C, Postma MJ, Hak E. Observed differences in invasive pneumococcal disease epidemiology after routine infant vaccination. Expert Rev Vaccines 2011;10:187-99.

5 French N, Gordon SB, Mwalukomo T, White SA, Mwafulirwa G, Longwe H, et al. A trial of a 7-valent pneumococcal conjugate vaccine in HIV-infected adults. N Engl J Med 2010;362:812-22.

6 Klugman KP, Madhi SA, Huebner RE, Kohberger R, Mbelle N, Pierce N. A trial of a 9-valent pneumococcal conjugate vaccine in children with and those without HIV infection. N Engl J Med 2003;349:1341-8.

7 Van Hoek AJ, Andrews N, Waight PA, Stowe J, Gates P, George R, et al. The effect of underlying clinical conditions on the risk of developing invasive pneumococcal disease in England. $J$ Infect 2012;65:17-24.

8 Miller E, Andrews NJ, Waight PA, Slack MP, George RC. Herd immunity and serotype replacement 4 years after seven-valent pneumococcal conjugate vaccination in England and Wales: an observational cohort study. Lancet Infect Dis 2011;11:760-8.

9 Jit M. The risk of sequelae due to pneumococcal meningitis in high-income countries: a systematic review and meta-analysis. J Infect 2010;61:114-24.

10 Howard LS, Sillis M, Pasteur MC, Kamath AV, Harrison BD. Microbiological profile of community-acquired pneumonia in adults over the last 20 years. $J$ Infect 2005;50:107-13.

11 Lim WS, Macfarlane JT, Boswell TC, Harrison TG, Rose D, Leinonen M, et al. Study of community acquired pneumonia aetiology (SCAPA) in adults admitted to hospital: implications for management guidelines. Thorax 2001;56:296-301.

12 Miller E, Andrews NJ, Waight PA, Slack MP, George RC. Effectiveness of the new serotypes in the 13-valent pneumococcal conjugate vaccine. Vaccine 2011;29:9127-31.

13 De Greeff SC, de Melker HE, Spanjaard L, Schouls LM, A van Deursen, SP van Mens, et al. Impact of seven-valent pneumococcal conjugate vaccine on the incidence and serotype distribution of invasive pneumococcal disease in the Netherlands. Poster serotype distribution of invasive pneumococcal disease in the Netherlands. Poster
presented at the European Society for Paediatric Infectious Diseases, June 2011. 2011. The Hague, Netherlands.

14 European Medicines Agency. Assessment report for Prevenar 13. 2010. www.ema.europa. eu/humandocs/Humans/EPAR/Prevenar13/Prevenar13.htm.

15 O'Hagen A, Buck C, Daneshkhah A, Eiser JR, Garthwaite PH, Jenkinson D. Uncertain judgements: eliciting experts' probabilities. Wiley, 2006.

16 Andrews N, Kaye P, Slack M, George R, Miller E. Effectiveness of the 13 valent pneumococcal conjugate vaccine against IPD in England and Wales. 2012. www2.kenes. com/ISPPD/Scientific/Documents/FinalAbstractbook.pdf.

17 Postma MJ. Public health economics of vaccines in the Netherlands: methodological issues and application. J Public Health 2008;16: 267-73.

18 Melegaro A, Choi YH, George R, Edmunds WJ, Miller E, Gay NJ. Dynamic models of pneumococcal carriage and the impact of the Heptavalent Pneumococcal Conjugate Vaccine on invasive pneumococcal disease. BMC Infect Dis 2010;10:90.

19 Office for National Statistics. 2011. www.statistics.gov.uk.

20 Bennett JE, Sumner W, Downs SM, Jaffe DM. Parents' utilities for outcomes of occult bacteremia. Arch Pediatr Adolesc Med 2000;154:43-48.

21 Rozenbaum MH, Sanders EA, van Hoek AJ, Jansen AG, van der Ende A, van den Dobbelsteen G, et al. Cost effectiveness of pneumococcal vaccination among Dutch infants: economic analysis of the seven valent pneumococcal conjugated vaccine and forecast for the 10 valent and 13 valent vaccines. BMJ 2010;340:c2509.

22 Oostenbrink R, A Moll HA, Essink-Bot ML. The EQ-5D and the Health Utilities Index for permanent sequelae after meningitis: a head-to-head comparison. J Clin Epidemio 2002;55:791-9.

23 Curtis L. Unit costs of health and social care. Personal Social Services Research Unit, 2010.

24 Melegaro A, Edmunds WJ. Cost-effectiveness analysis of pneumococcal conjugate vaccination in England and Wales. Vaccine 2004;22:4203-14.

25 National Institute for Health and Clinical Excellence. Updated guide to the methods of technology appraisal—June 2008. NICE, 2008. 


\section{What is already known on this topic}

Although vaccination programmes with the 23 valent polysaccharide vaccine are in place, pneumococcal infections still disproportionately affect people with certain high risk conditions

New conjugated pneumococcal vaccines have been shown to be highly effective in preventing vaccine type disease in infants and people infected with HIV

Recently the European Commission extended the indication of the 13 valent pneumococcal conjugate vaccine to adults aged 50 and older

\section{What this study adds}

Infant vaccination with the 13 valent pneumococcal conjugate vaccine is likely to reduce the incidence in the whole population, thereby reducing the added benefit (and cost effectiveness) of targeting at risk groups

Whether targeting high risk groups is cost effective also depends on how effective the vaccine is at protecting high risk people against non-bacteraemic pneumococcal pneumonia

Delaying a decision until such efficacy data become available may render a targeted policy less cost effective if infant vaccination has already been introduced

26 Department of Health. The green book. Immunisation against infectious disease. 2011. http://webarchive.nationalarchives.gov.uk/+/www.dh.gov.uk/prod_consum_dh/groups/dh digitalassets/@dh/@en/documents/digitalasset/dh_122639.pdf.

27 Begun F, Pebody R. Seasonal influenza vaccine uptake among the 65 years and over and under 65 years at risk in England. Winter season 2009-10. 2010. www.dh.gov.uk prod_consum_dh/groups/dh_digitalassets/@dh/@en/@ps/documents/digitalasset/dh_ 118645.pdf.

28 Hak E, Sanders EA, Verheij TJ, Huijts SM, Gruber WC, Tansey S, et al. Rationale and design of CAPITA: a RCT of 13-valent conjugated pneumococcal vaccine efficacy among older adults. Neth J Med 2008;66:378-83.

29 Choi YH, Jit M, Gay N, Andrews N, Waight PA, Melegaro A, et al. 7-valent pneumococcal conjugate vaccination in England and wales: is it still beneficial despite high levels of serotype replacement? PLoS One 2011;6:e26190.

30 Brueggemann AB, Griffiths DT, Meats E, Peto T, Crook DW, Spratt BG. Clonal relationships between invasive and carriage Streptococcus pneumoniae and serotype- and clone-specific differences in invasive disease potential. $J$ Infect Dis 2003;187:1424-32.

31 Trotter CL, Waight P, Andrews NJ, Slack M, Efstratiou A, George R, et al. Epidemiology of invasive pneumococcal disease in the pre-conjugate vaccine era: England and Wales, 1996-2006. J Infect 2010;60:200-8.

32 Flasche S, van Hoek AJ, Sheasby E, Waight P, Andrews N, Sheppard C, et al. Effect of pneumococcal conjugate vaccination on serotype-specific carriage and invasive disease in England: a cross-sectional study. PLoS Med 2011;8:e1001017.

33 Rozenbaum MH, Hak E, van der Werf TS, Postma M.J. Results of a cohort model analysis of the cost-effectiveness of routine immunization with 13-valent pneumococcal conjugate vaccine of those aged $>$ or $=65$ years in the Netherlands. Clin Ther 2010;32:1517-32.

34 Smith KJ, Wateska AR, Nowalk MP, Raymund M, Nuorti JP, Zimmerman RK. Cost-effectiveness of adult vaccination strategies using pneumococcal conjugate vaccine compared with pneumococcal polysaccharide vaccine. JAMA 2012;307:804-12.

35 Drummond MF, Jefferson TO. Guidelines for authors and peer reviewers of economic submissions to the BMJ. The BMJ Economic Evaluation Working Party. BMJ 1996;313:275-83.
36 Zakikhany K, Degail MA, Lamagni T, Waight $\mathrm{P}$, Guy R, Zhao H, et al. Increase in invasive Streptococcus pyogenes and Streptococcus pneumoniae infections in England, December 2010 to January 2011. Euro Surveill 2011;16:ii19785.

37 Joint committee on vaccination and immunization. Minute of the meeting on Wednesday 13 June 2012. www.wp.dh.gov.uk/transparency/files/2012/07/JCVI-minutes-13-June2012-draft.pdf.

38 Impfplan Österreich 2012. www.bmg.gv.at/home/Schwerpunkte/Praevention/Impfen/ Oesterreichischer_Impfplan_2012.

39 http://static.diavgeia.gov.gr/doc/45 $\Psi \Psi \Theta-2 \Theta M$. [Greek]2012.

40 Haut conseil de la Sante Publique. 2012. www.hcsp.fr/explore.cgi/avisrapports? ae=avisrapports \&menu $=09$

41 Empfehlungen der Sächsischen Impfkommission zur Durchführung von Schutzimpfungen im Freistaat Sachsen [German]. 2012. www.slaek.de/60infos/infosarzt/36impfen/pdt/e1. pdf.

42 British Medical Association, Royal Pharmaceutical Society of Great Britain. British national formulary. BMA, RPS, 2010. (No 60).

43 Nakagawa F, Lodwick RK, Smith CJ Smith R, Cambiano V Lundgren JD, et al. Projected life expectancy of people with HIV according to timing of diagnosis. AIDS 2012;26:335-43.

44 Harrison KM, Song R, Zhang X. Life expectancy after HIV diagnosis based on national HIV surveillance data from 25 states, United States. J Acquir Immune Defic Syndr 2010;53:124-30

\section{Accepted: 27 September 2012}

\section{Cite this as: BMJ 2012;345:e6879}

This is an open-access article distributed under the terms of the Creative Commons Attribution Non-commercial License, which permits use, distribution, and reproduction in any medium, provided the original work is properly cited, the use is non commercial and is otherwise in compliance with the license. See: $\mathrm{http}: / /$ creativecommons.org/licenses/bync/2.0/ and http://creativecommons.org/licenses/by-nc/2.0/legalcode. 


\section{Tables}

\section{Table 1| Variables used in economic model}

\begin{tabular}{|c|c|c|c|}
\hline Variables & Expected value & Distribution & Reference \\
\hline Age specific incidence & $\begin{array}{l}\text { See appendix } 8 \text { in supplementary } \\
\text { file }\end{array}$ & NA & See methods \\
\hline Odds of IPD* & Age and risk group dependent ${ }^{\star}$ & Log normal & 7 , see methods \\
\hline Case fatality ratio $\dagger$ & Age and risk group dependent $†$ & $\beta$ & 7, see methods \\
\hline Share of meningitis in total burden of IPD & 3-8\% (age dependent) & Fixed & See methods \\
\hline Share of empyema in total burden of IPD & $1-5 \%$ (age dependent) & Fixed & See methods \\
\hline \multicolumn{4}{|l|}{ Vaccine efficacy against IPD $\ddagger$} \\
\hline \multicolumn{4}{|l|}{ High risk immunocompetent: } \\
\hline$<65$ years & 0.71 & $\beta(\alpha 2.1, \beta 0.863)$ & See methods \\
\hline$\geq 65$ years & 0.63 & $\beta(a 2.01, \beta 1.19)$ & See methods \\
\hline \multicolumn{4}{|l|}{ High risk immunocompromised: } \\
\hline$<65$ years & 0.53 & $\beta(a 1.59, \beta 1.41)$ & See methods \\
\hline$\geq 65$ years & 0.43 & $\beta(\alpha 1.21, \beta 1.62)$ & See methods \\
\hline \multicolumn{4}{|c|}{ Vaccine efficacy against non-bacteraemic pneumococcal pneumoniał } \\
\hline \multicolumn{4}{|l|}{ High risk immunocompetent: } \\
\hline$<65$ years & 0.46 & $\beta(a 1.88, \beta 2.19)$ & See methods \\
\hline$\geq 65$ years & 0.40 & $\beta(a$ 1.47, $\beta 2.2)$ & See methods \\
\hline \multicolumn{4}{|l|}{ High risk immunocompromised: } \\
\hline$<65$ years & 0.33 & $\beta(\alpha 1.24, \beta 2.55)$ & See methods \\
\hline$\geq 65$ years & 0.27 & $\beta(a 1.27, \beta 3.47)$ & See methods \\
\hline \multicolumn{4}{|l|}{ Waning immunity (per year)§ } \\
\hline \multicolumn{4}{|l|}{ Immunocompetent: } \\
\hline$<65$ years & 0.11 & See methods & See methods \\
\hline$\geq 65$ years & 0.25 & See methods & See methods \\
\hline \multicolumn{4}{|l|}{ Immunocompromised: } \\
\hline$<65$ years & 0.24 & See methods & See methods \\
\hline$\geq 65$ years & 0.26 & See methods & See methods \\
\hline \multicolumn{4}{|l|}{ Prevalence of sequelae after meningitis } \\
\hline Deafness & 0.08 & $\beta$ (mean 0.08 SE 0.03) & 9 \\
\hline Mild hearing loss & 0.21 & $\beta$ (mean 0.21 SE 0.02) & 9 \\
\hline Seizures and hydrocephalus & 0.07 & $\beta$ (mean 0.07 SE 0.02) & 9 \\
\hline Spasticity or paresis & 0.09 & $\beta$ (mean 0.09 SE 0.01) & 9 \\
\hline Cranial nerve palsy & 0.12 & $\beta$ (mean 0.12 SE 0.04) & 9 \\
\hline \multicolumn{4}{|l|}{ Quality adjusted life year losses } \\
\hline Hospital admission for meningitis & 0.023 & $\beta$ (mean 0.023 SE 0.031) & 2120 \\
\hline Hospital admission for bacteraemia & 0.0079 & $\beta$ (mean 0.079 SE 0.083) & 21 \\
\hline Hospital admission for non-bacteraemic pneumonia & 0.006 & Normal (mean 0.006 SD 0.0015) & 2120 \\
\hline \multicolumn{4}{|l|}{ Quality of life weights } \\
\hline Deafness & 0.81 & $\beta$ (mean 0.81 SE 0. 028) & 22 \\
\hline Mild hearing loss & 0.91 & $\beta$ (mean 0.91 SE 0.015) & 22 \\
\hline Seizures & 0.83 & $\beta$ (mean 0.83 SE 0.015) & 22 \\
\hline Hydrocephalus & 0.62 & $\beta$ (mean 0.62 SE 0.021) & 22 \\
\hline Spasticity or paresis & 0.67 & $\beta$ (mean 0.67 SE 0.023) & 22 \\
\hline Cranial nerve palsy & 0.67 & $\beta$ (mean 0.67 SE 0.023) & 22 \\
\hline \multicolumn{4}{|l|}{ Costs $(£)$} \\
\hline Case of meningitis ${ }^{* *}$ & 6509 & Normal (mean 6509 SD 405) & See methods \\
\hline
\end{tabular}




\section{Table 1 (continued)}

\begin{tabular}{|c|c|c|c|}
\hline Variables & Expected value & Distribution & Reference \\
\hline Case of empyema** & 7538 & Normal (mean 7665 SD 444) & See methods \\
\hline Short hospital stay for other IPD** & 825 & Normal (mean 839 SD 3.93) & See methods \\
\hline \multicolumn{4}{|l|}{ Long hospital stay for other IPD: } \\
\hline With excess days in hospital ${ }^{* *}$ & 8977 & Normal (mean 9129 SD 142) & See methods \\
\hline Without excess days in hospital ${ }^{* *}$ & 3022 & Normal (mean 3073 SD 19) & See methods \\
\hline Admitted to hospital for pneumonia & 661 & Normal (mean 672 SD 168) & See methods \\
\hline Chance of long stay for IPD & 0.61 & $\beta(\alpha 5075 \beta$ 8257) & See methods \\
\hline Chance of excess days during long stay for IPD & 0.46 & $\beta(\alpha 2328 \beta 5075)$ & See methods \\
\hline \multicolumn{4}{|l|}{ Lifetime costs after meningitis: } \\
\hline In first year & 6591 & Log normal (mean 8.7 SD 0.4) & 24 \\
\hline In subsequent years & 203 & Log normal (mean 8.7 SD 0.4) & 24 \\
\hline Outpatient follow-up for meningitis & 382 & Log normal (mean 5.2 SD 0.4) & 24 \\
\hline Cost of 13 valent pneumococcal conjugate vaccine & 49.10 & Fixed & 42 \\
\hline Administration costs & 7.51 & Fixed & 42 \\
\hline \multicolumn{4}{|l|}{ Other variables } \\
\hline Herd effect due to infant vaccination & $\begin{array}{l}\text { See appendix } 2 \text { in supplementary } \\
\text { file }\end{array}$ & Normal & $\begin{array}{l}\text { See appendix } 2 \text { in supplementary } \\
\text { file }\end{array}$ \\
\hline Life expectancy among high risk groups & $\begin{array}{l}\text { See appendix } 2 \text { in supplementary } \\
\text { file }\end{array}$ & NA & See methods \\
\hline Discount rate for costs and health effects & $3.5 \%$ & NA & 25 \\
\hline
\end{tabular}

IPD=invasive pneumococcal disease; $£ 1.00(\$ 1.6 ; € 1.2)$.

*Odds ratio of IPD comparing risk groups to non-risk groups. Specific odds ratios can be found in Van Hoek et al. ${ }^{7}$

†Age specific case fatality ratios can be found in Van Hoek et al. ${ }^{7}$

$\ddagger$ After single dose during first year of vaccination. Efficacy estimates do not apply for serotype 3 (see method section). ${ }^{16}$ Estimates of vaccine efficacy after two doses are listed in appendix 4 in the supplementary file.

$\S$ Annual waning factor was calculated by using the experts' estimation of vaccine efficacy during first and third year after vaccination using annual exponential decay of immunity.

TSame quality of life year decrement was assumed for invasive pneumonia, bacteraemia with focus, and bacteraemia without focus.

${ }^{* \star}$ Mean costs were sampled from a normal distribution with a mean equal to the log normal mean and standard deviation equal to the standard error of the log normal mean. 
Table 2| Total burden of invasive pneumococcal disease (IPD) due to vaccine serotype (undiscounted) over nine year period (2012-13 to 2020-21) in people at high risk

\section{Variables}

Cases of IPD due to

Without high risk vaccination and without herd protection benefits of 13 valent pneumococcal conjugate vaccine serotypes

\begin{tabular}{|c|c|c|c|c|}
\hline Cases prevented by herd effects of infant 13 valent pneumococcal conjugate vaccine programme $\dagger$ & 6189 & 1538 & 28397 & 30382 \\
\hline $\begin{array}{l}\text { Without high risk vaccination and with herd effects of additional six serotypes in } 13 \text { valent pneumococcal } \\
\text { conjugate vaccine }\end{array}$ & 1333 & 357 & 5854 & 6197 \\
\hline With high risk group vaccination (including herd effects of infant programme) $\ddagger$ & 927 & 247 & 4033 & 4274 \\
\hline Averted burden by high risk vaccination (incremental effects)§ & 406 & 110 & 1821 & 1923 \\
\hline
\end{tabular}

QALYs=quality adjusted life years.

*Only including herd effect due to serotypes included in seven valent pneumococcal conjugate vaccine (excluding herd effect due to six additional serotypes included in 13 valent pneumococcal conjugate vaccine. †Herd effects due to additional six serotypes in 13 valent pneumococcal conjugate vaccine based on incidence after vaccination with seven valent pneumococcal conjugate vaccine (see methods and appendix 2 in supplementary file).

$\ddagger$ Vaccination uptake to be assumed similar to that of annual influenza uptake (see methods).

§Numbers may not add up owing to rounding. 
Table 3| Budget impact (total costs) of vaccinating different risk groups ( $(\mathrm{m})$ with 13 valent pneumococcal conjugate vaccine according to assumed uptakes

\begin{tabular}{lccc} 
& \multicolumn{2}{c}{ Assumed uptake $^{\star}$} & \\
\cline { 2 - 4 } Risk group & $\begin{array}{c}\text { Similar to influenza programme (base } \\
\text { case) }\end{array}$ & $\mathbf{8 0 \%}$ & $\begin{array}{c}\text { Similar to annual 23 valent } \\
\text { polysaccharide vaccination programmet }\end{array}$ \\
Any risk group & 233 & 290 & 17.8 \\
\hline Splenic dysfunction & 6.3 & 8.9 & 0.35 \\
\hline Chronic respiratory disease & 34.1 & 41.5 & 2.80 \\
\hline Chronic heart disease & 116 & 1411 & 9.60 \\
\hline Chronic kidney disease & 71.5 & 83.4 & 6.40 \\
\hline Chronic liver disease & 4.64 & 6.4 & 0.24 \\
\hline Diabetes & 59.2 & 75.2 & 4.15 \\
\hline Immunocompromised & 17.9 & 24.0 & 1.12 \\
\hline Infected with HIV & 0.37 & 0.54 & 0.01
\end{tabular}

$£ 1.00(\$ 1.6 ; € 1.2)$.

${ }^{*}$ Annual influenza coverage $34.5 \%$ in 2-15 year olds, $53.6 \%$ in $16-65$ year olds, and $72.4 \%$ in those aged $\geq 65$ years. ${ }^{27}$ Sum of costs of separate risk groups are higher than total costs of any risk group as people may have more than one underlying condition.

†Annual uptake $4.1 \%$ in $2-15$ year olds, $1.5 \%$ in $16-65$ year olds, and $7.2 \%$ in those aged $\geq 65$ years). See appendix 7 in supplementary file. 
Table 4| Incremental cost effectiveness ratios (ICERs) in £/quality adjusted life year (QALY) per risk group assuming vaccination will be introduced in epidemiological year 2012-13

\begin{tabular}{lcc} 
& & ICER (E/QALY) \\
\cline { 2 - 3 } Risk group & Base case* & $\begin{array}{c}\text { Including non-bacteraemic pneumococcal } \\
\text { pneumonia }\end{array}$ \\
Any risk group & 183680 & 17503 \\
\hline Splenic dysfunction & 1204091 & 37686 \\
\hline Chronic respiratory disease & 90243 & 14832 \\
\hline Chronic heart disease & 161063 & 16043 \\
\hline Chronic kidney disease & 493682 & 22641 \\
\hline Chronic liver disease & 20324 & 10825 \\
\hline Diabetes & 269750 & 18459 \\
\hline Immunocompromised & 90720 & 24296 \\
\hline Infected with HIV $\dagger$ & 61239 & 28144 \\
\hline
\end{tabular}

$£ 1.00(\$ 1.6 ; € 1.2)$.

*Assuming no overall impact on non-bacteraemic pneumonia in high risk group.

†When the assumption was made that life expectancy of people infected with HIV would be similar to high risk immonocompetent people, ${ }^{434}$ incremental cost effectiveness ratios were estimated at £54 409/QALY in base case analysis and at £25 717/QALY when an effect against non-bacteraemic pneumococcal pneumonia was included. 
Table 5| Result of scenario analyses on incremental cost effectiveness ratio (£/quality adjusted life year, QALY) for those risk groups that had an incremental cost effectiveness ratio (ICER) $<100000$ per QALY in base case for epidemiological year 2012-13

\begin{tabular}{|c|c|c|c|c|c|}
\hline Variables & Any risk group & $\begin{array}{l}\text { Chronic respiratory } \\
\text { disease }\end{array}$ & Chronic liver disease & Immunocompromised & Infected with HIV \\
\hline Base case & 183680 & 90243 & 20324 & 90720 & 61239 \\
\hline $\begin{array}{l}\text { No herd effects due to any serotypes in } \\
13 \text { valent pneumococcal conjugate } \\
\text { vaccine* }^{*}\end{array}$ & 37687 & 18061 & 2848 & 20059 & 10059 \\
\hline $\begin{array}{l}\text { No herd effects due to six additional } \\
\text { serotypes in } 13 \text { valent pneumococcal } \\
\text { conjugate vaccine } \dagger\end{array}$ & 46903 & 22715 & 3529 & 25259 & 12404 \\
\hline $\begin{array}{l}\text { No herd effects due to serotypes } 1 \text { and } \\
5 \dagger\end{array}$ & 74882 & 36122 & 6496 & 41115 & 25181 \\
\hline $\begin{array}{l}\text { Herd effect of infant } 13 \text { valent } \\
\text { pneumococcal conjugate vaccine delayed } \\
\text { by two years }\end{array}$ & 128603 & 63257 & 13369 & 63301 & 39452 \\
\hline Vaccine price $25 \%$ reduced & 143564 & 70390 & 15772 & 70720 & 47342 \\
\hline $\begin{array}{l}\text { Vaccine price } 25 \% \text { reduced and no } \\
\text { administration costs }\end{array}$ & 119021 & 58244 & 12987 & 58484 & 38840 \\
\hline No waning immunity & 141999 & 69927 & 17013 & 65107 & 45181 \\
\hline No discounting & 120495 & 60164 & 11570 & 59730 & 34484 \\
\hline Life expectancy of normal population & 163070 & 79937 & 18446 & 81036 & 50331 \\
\hline Double vaccine dose & 308886 & 153053 & 34429 & 143581 & 97066 \\
\hline $\begin{array}{l}15 \% \text { higher incidence of invasive } \\
\text { pneumococcal disease }\end{array}$ & 159550 & 78302 & 17586 & 78691 & 52880 \\
\hline $\begin{array}{l}\text { Assuming } 13 \text { valent pneumococcal } \\
\text { conjugate vaccine to be effective against } \\
\text { serotype } 3\end{array}$ & 150326 & 73331 & 17620 & 74099 & 54099 \\
\hline
\end{tabular}

*No further reduction as from 2009-10 for all serotypes included in 13 valent pneumococcal conjugate vaccine.

$\nmid 20 \%$ less herd effects could be achieved when serotypes 1 and 5 were not assumed to provide herd protection and $80 \%$ less herd effects could be achieved when six additional serotypes included in 13 valent pneumococcal conjugate vaccine would not provide any herd effect compared with maximum herd effect (for example, total eradication of all serotypes included in 13 valent pneumococcal conjugate vaccine) calculated by using specific incidence data on serotype for 2009-10 and projecting forward. 


\section{Figures}

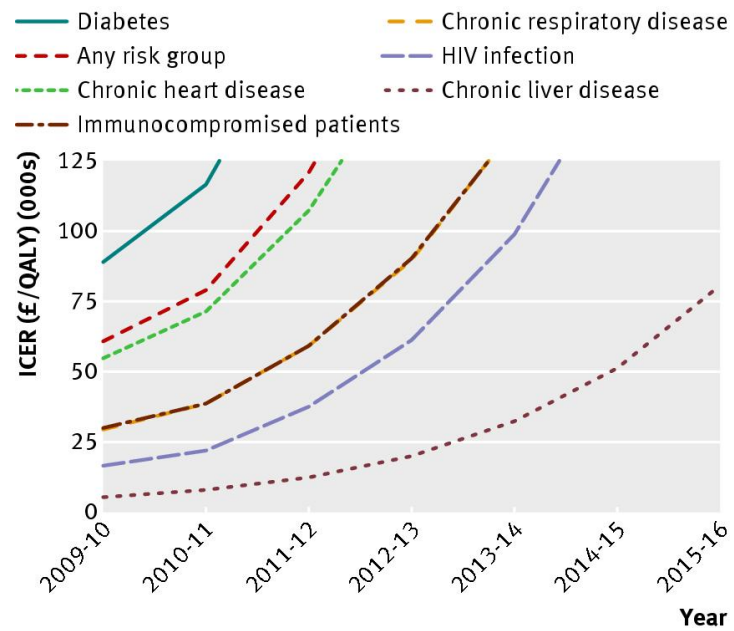

Fig 1 Impact of time on incremental cost effectiveness ratio. QALY=quality adjusted life year

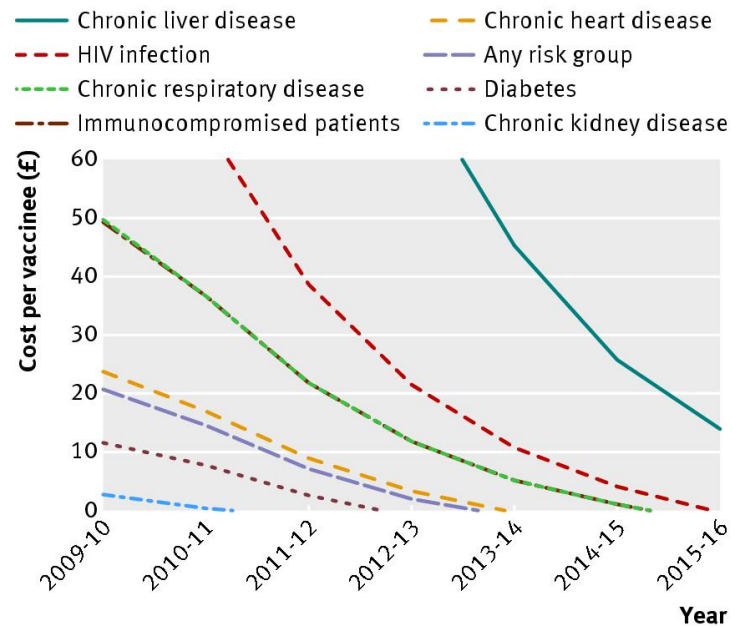

Fig 2 Maximum costs per vaccinee (including costs of vaccine and administration) to consider risk group vaccination cost effective (incremental cost effectiveness ratio of $\leq £ 30000$ per quality adjusted life year) 

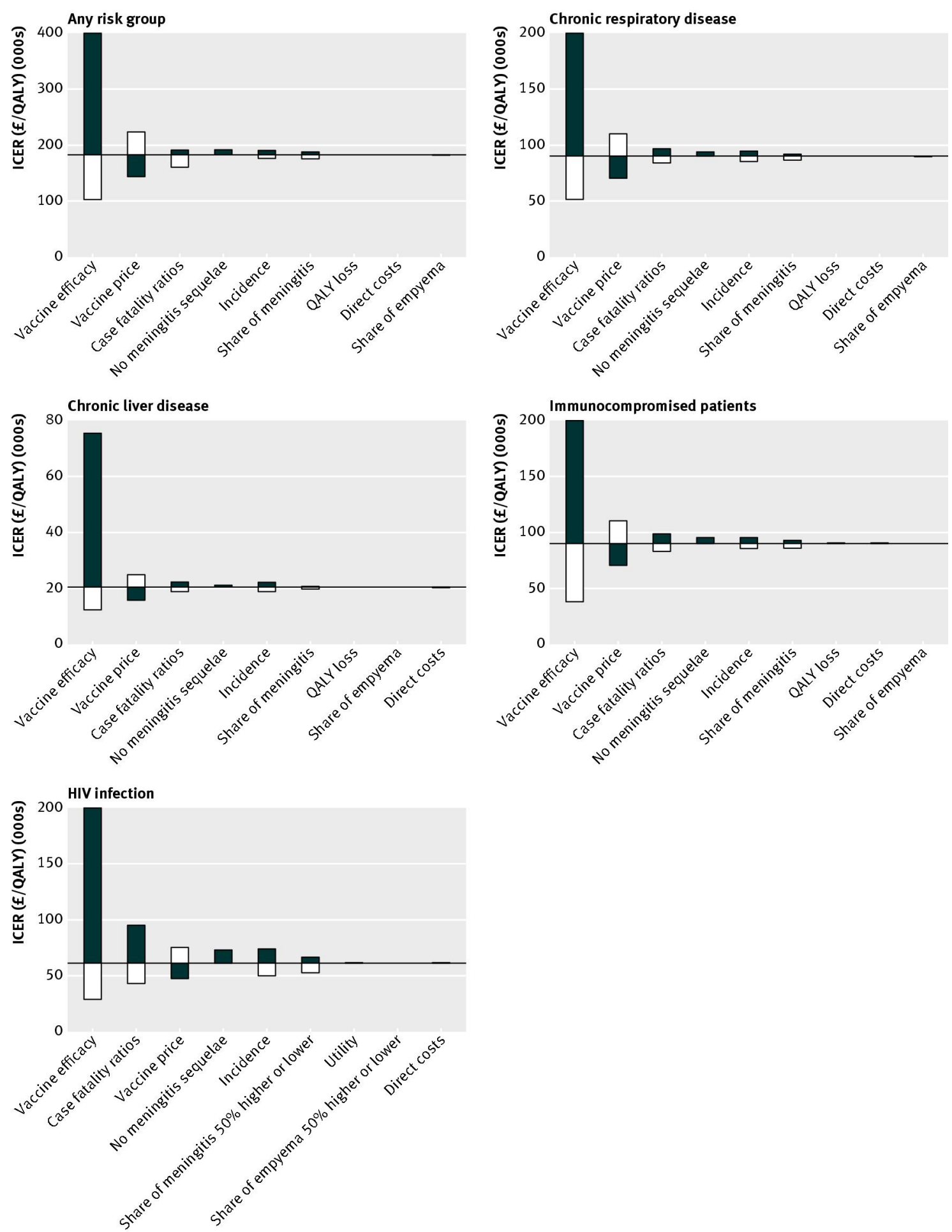

Fig 3 Univariate sensitivity analysis for any at risk group. Variables were changed over their $5 \%$ and $95 \%$ quantiles, with exception of share of meningitis and empyema, which were varied by $50 \%$. Incidence was altered by varying odds of invasive pneumococcal disease in those with risk factors compared with those without. Bar for lower vaccine efficacy are cut-off for all at risk groups except immunocompromised patients. Please note that the scales of the figures vary. QALY=quality adjusted life year 
Chronic liver disease

- - Chronic respiratory disease (with pneumonia)

---- Any risk group (with pneumonia)

-- Chronic liver disease (with pneumonia)

- - Immunocompromised patients (with pneumonia)

- - HIV infection (with pneumonia)

.... HIV infection

-.-- Chronic respiratory disease

- - Immunocompromised patients

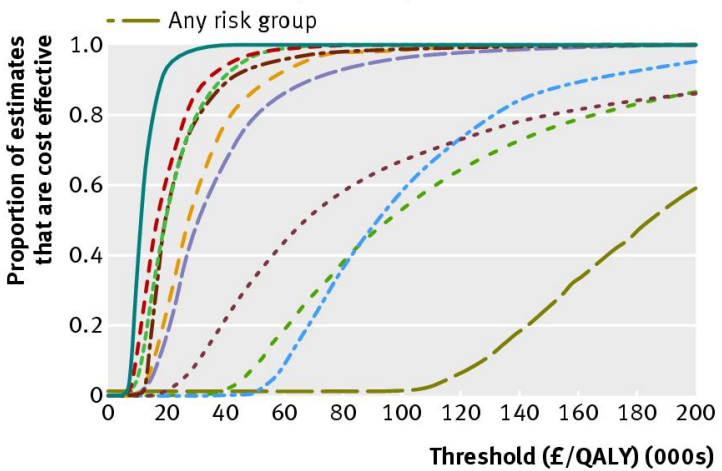

Fig 4 Results of probabilistic sensitivity analysis 